PROCEEDINGS OF THE

AMERICAN MATHEMATICAL SOCIETY

Volume 130, Number 3, Pages 619-624

S 0002-9939(01)06090-7

Article electronically published on June 19, 2001

\title{
EVERY DIASSOCIATIVE A-LOOP IS MOUFANG
}

\author{
MICHAEL K. KINYON, KENNETH KUNEN, AND J. D. PHILLIPS
}

(Communicated by Lance W. Small)

\begin{abstract}
An A-loop is a loop in which every inner mapping is an automorphism. A problem which had been open since 1956 is settled by showing that every diassociative A-loop is Moufang.
\end{abstract}

\section{INTRODUCTION}

A loop $(L, \cdot)$ consists of a nonempty set $L$ with a binary operation $\cdot$ on $L$ such that (i) given $a, b \in L$, the equations $a x=b$ and $y a=b$ each have unique solutions $x, y \in L$, and (ii) there exists an identity element $1 \in L$ satisfying $1 x=x 1=x$ for all $x \in L$. As usual, we abbreviate the binary operation by juxtaposition. Two varieties of loops which have been widely discussed in the literature are the Moufang loops and the A-loops.

A Moufang loop is a loop satisfying the identity

$$
x(y \cdot x z)=(x y \cdot x) z .
$$

These were introduced by R. Moufang in 1934 [14, and are discussed in detail in the texts by Bruck [1] and Pflugfelder [17]. By Moufang's Theorem (11, VII.4; [17, IV.2.9), every Moufang loop is diassociative; that is, the subloop $\langle x, y\rangle$ generated by any pair of elements is a group.

For $x \in L$, the left and right translations by $x$ are defined by $y L(x)=x y$ and $y R(x)=y x$, respectively. The multiplication group of $L$ is the permutation group $\operatorname{Mlt}(L)=\langle R(x), L(x): x \in L\rangle$ generated by all left and right translations. The inner mapping group is the subgroup $\operatorname{Mlt}_{1}(L)$ fixing 1 . If $L$ is a group, then $\operatorname{Mlt}_{1}(L)$ is the group of inner automorphisms of $L$.

In 1956, R.H. Bruck and L.J. Paige 2 defined an A-loop to be a loop in which every inner mapping is an automorphism. Many of the basic theorems about Aloops are contained in [2] for example, A-loops are always power associative (every $\langle x\rangle$ is a group), but not necessarily diassociative. In the same paper, Bruck and Paige included a detailed study of the diassociative A-loops, pointing out that these satisfy "many of the properties of Moufang loops". In hindsight, this is not surprising, since, as we will show:

Theorem 1. Every diassociative A-loop is a Moufang loop.

Received by the editors August 3, 2000 and, in revised form, August 18, 2000.

2000 Mathematics Subject Classification. Primary 20N05; Secondary 68T15.

Key words and phrases. Diassociative loop, A-loop, Moufang loop.

The second author was partially supported by NSF Grant DMS-9704520.

(C)2001 American Mathematical Society 
For commutative loops, this was proved in 1958 by J.M. Osborn [16]. Conversely, every commutative Moufang loop is an A-loop (see Bruck [1], Lemma VII.3.3). However, not all Moufang loops are A-loops; 2, 18], together with the results of the present paper, provide a simple description of the diassociative A-loops as a sub-variety of the Moufang loops (see Corollary 22). Further work on A-loops is contained in 4, 5].

By our Theorem 1, we have:

Corollary 1. For an A-loop, the following are equivalent:

1. L has the inverse property, i.e., $x^{-1}(x y)=y$ and $(x y) y^{-1}=x$ for all $x, y \in L$;

2. $L$ has the alternative property, i.e., $x(x y)=x^{2} y$ and $(x y) y=x y^{2}$ for all $x, y \in L$;

3. $L$ is diassociative.

4. $L$ is a Moufang loop.

The equivalence of the first three items is from Bruck and Paige [2], Theorem 3.1. (One may begin with even weaker hypotheses, but we will not pursue this here.)

In any loop, the inner mapping group $\operatorname{Mlt}_{1}(L)$ is generated by the left, right, and middle inner mappings defined, respectively, by:

$$
\begin{aligned}
L(x, y) & =L(x) L(y) L(y x)^{-1}, \\
R(x, y) & =R(x) R(y) R(x y)^{-1}, \\
T(x) & =R(x) L(x)^{-1}
\end{aligned}
$$

([1], IV.1, 17], I.5.2). Bruck and Paige ([2], (3.42)) showed that diassociative Aloops satisfy

$$
[R(x, y) R(y, x)]^{-1} T(x) T(y)=T(x y) .
$$

Furthermore, they showed (see Corollary on p. 315) that for Moufang A-loops, the map $T: L \rightarrow \operatorname{Mlt}_{1}(L)$ (where $x \mapsto T(x)$ ) is a homomorphism (i.e., $T(x) T(y)=$ $T(x y)$, so $\left.R(x, y)=R(y, x)^{-1}\right)$. Not surprisingly, one of our key lemmas will be:

Lemma 1. If $L$ is a diassociative $A$-loop, then $T: L \rightarrow \operatorname{Mlt}_{1}(L)$ is a homomorphism.

The nucleus, $\operatorname{Nuc}(L)$, of an inverse property loop $L$ is the normal subloop of all elements that associate with all pairs of elements from $L$, i.e., $\operatorname{Nuc}(L)=\{x \in L$ : $(x y) z=x(y z)$ for all $y, z \in L\}$. By results already in the literature, we have the following corollary to Theorem[1:

Corollary 2. $L$ is a diassociative A-loop if and only if $L$ is Moufang and $L / \operatorname{Nuc}(L)$ is a commutative loop of exponent three.

Proof. In any Moufang loop, each $T(x)$ is a pseudo-automorphism with companion $x^{-3}$, and each $R(x, y)=L\left(x^{-1}, y^{-1}\right)$ is a pseudo-automorphism with companion the commutator $(x, y)$ ([1], Lemma VII.2.2). In general, if $c$ is a companion of the pseudo-automorphism $\varphi$, then $c$ is in the nucleus iff $\varphi$ is an automorphism. Thus all cubes and commutators are in the nucleus iff all inner mappings are automorphisms.

Every Moufang A-loop is an $M_{4}$ loop in the terminology of Pflugfelder [15, 17]; that is, it satisfies the identity $(x y)\left(z x^{4}\right)=(x \cdot y z) x^{4}$ (since cubes are in the nucleus and $(x y)(z x)=(x \cdot y z) x$ is a Moufang identity). We do not know whether an $M_{4}$ 
loop $L$ must be an A-loop. By [15], Theorem 1, $L$ is Moufang and $L / \operatorname{Nuc}(L)$ has exponent three, but it is not clear whether $L / \operatorname{Nuc}(L)$ is necessarily commutative. We also do not know whether every loop isotope of a Moufang A-loop is a (Moufang) A-loop. This would be true if every $M_{4}$ loop is an A-loop, since the $M_{k}$ loops are isotopically invariant (15], Theorem 2; 17], IV.4.12).

Our investigations were aided by the automated deduction tool OTTER developed by McCune [12; see Section 4 for further discussion.

\section{Preliminaries}

In preparation for the proofs of Lemma 1 and Theorem 1, we now establish some notation and recall some basic results from 2. Let $L$ be a diassociative A-loop. One can then derive many equations relating the $L(x, y), R(x, y)$, and $T(x)$.

Define the permutation $J$ of $L$ by $x J=x^{-1}$. Conjugating by $J$, we have $R(x)^{J}=$ $J R(x) J=L\left(x^{-1}\right)$; likewise, $L(x)^{J}=R\left(x^{-1}\right)$ and $L(x, y)^{J}=R\left(x^{-1}, y^{-1}\right)$. Note that $\varphi^{J}=\varphi$ for all automorphisms $\varphi$ of $L$; in particular, for all $\varphi \in \operatorname{Mlt}_{1}(L)$. Taking $\varphi=L(x, y)$, we have

$$
L(x, y)=R\left(x^{-1}, y^{-1}\right)
$$

for $x, y \in L$. Furthermore, from [2] ((3.31) and (3.32)), we have the following formulas for the inverses of the right and left inner mappings:

$$
\begin{aligned}
& R(x, y)^{-1}=R\left(y^{-1}, x^{-1}\right), \\
& L(x, y)^{-1}=L\left(y^{-1}, x^{-1}\right) .
\end{aligned}
$$

The fact that each $T(x)$ is an automorphism immediately implies:

$$
\begin{aligned}
& R(y) T(x)=T(x) R\left(x^{-1} y x\right), \\
& L(y) T(x)=T(x) L\left(x^{-1} y x\right) .
\end{aligned}
$$

Another useful inner mapping is defined by

$$
C(x, y)=R(x) L(y) R\left(x^{-1}\right) L\left(y^{-1}\right) .
$$

Since $C(x, y)^{J}=C(x, y)$, we also have

$$
C(x, y)=L\left(x^{-1}\right) R\left(y^{-1}\right) L(x) R(y) .
$$

Also, by [2] (3.41),

$$
C(x, y)=R(x, y) R(y, x)^{-1} .
$$

Further equations relating the $C(x, y), R(x, y), L(x, y)$ will be proved later (see Corollaries 3 and (4). As pointed out in 2], in any loop, if $\varphi$ is an automorphism which fixes an element $p$, then $\varphi$ commutes with $L(p)$ and $R(p)$. In particular ([2], Lemma 3.3(i,ii,iii)), if $p, q, r$ are contained in any subgroup of $L$, then

$$
\begin{aligned}
& R(p) R(q, r)=R(q, r) R(p) ; \quad L(p) R(q, r)=R(q, r) L(p), \\
& R(p) L(q, r)=L(q, r) R(p) ; \quad L(p) L(q, r)=L(q, r) L(p), \\
& R(p) C(q, r)=C(q, r) R(p) ; \quad L(p) C(q, r)=C(q, r) L(p) .
\end{aligned}
$$

One consequence is that the factors in the right and left inner mappings can by cyclically permuted:

$$
\begin{aligned}
& R(x, y)=R(y) R\left(y^{-1} x^{-1}\right) R(x)=R\left(y^{-1} x^{-1}\right) R(x) R(y), \\
& L(x, y)=L(y) L\left(x^{-1} y^{-1}\right) L(x)=L\left(x^{-1} y^{-1}\right) L(x) L(y) .
\end{aligned}
$$




\section{Proofs}

Proof of Lemma [1. For $x, y, z \in L$, we compute

$$
\begin{array}{rlrl}
z L(x y) T(x) & =y L(x) R(z) T(x) & & \\
& =y C\left(x^{-1}, z^{-1}\right) R(z) L(x) T(x) & & \text { by }(2.7) \\
& =y C\left(x^{-1}, z^{-1}\right) R(z) R(x) & & \\
& =y R(z) R(x) C\left(x^{-1}, z^{-1}\right) & & \text { by }(2.11) \\
& =y R(z) L\left(z^{-1}\right) R(x) L(z) & & \text { by }(2.6) \\
& =y T(z) R(x) L(z) . &
\end{array}
$$

By the mirror of this calculation and switching $x$ and $y$, we obtain

$$
z R(x y) T\left(y^{-1}\right)=x T\left(z^{-1}\right) L(y) R(z) .
$$

But by (2.4), we have

$$
y T(z) R(x) L(z)=y R\left(z x z^{-1}\right) R(z)=x T\left(z^{-1}\right) L(y) R(z) .
$$

Hence, $L(x y) T(x)=R(x y) T\left(y^{-1}\right)$, so that $T(x) T(y)=L(x y)^{-1} R(x y)=T(x y)$.

\section{Corollary 3.}

$$
\begin{aligned}
R(x, y)^{-1} & =R(y, x), \\
R(x, y) & =R\left(x^{-1}, y^{-1}\right)=L(x, y)=L\left(x^{-1}, y^{-1}\right), \\
C(x, y) & =C\left(x^{-1}, y^{-1}\right)=R(x, y)^{2} .
\end{aligned}
$$

Proof. (3.1) follows from Lemma 1 and (1.2). To get (3.2), apply (2.2) and (2.1). Then, (3.3) follows by using (2.8).

Lemma 2. For all $x, y, z$ in a diassociative A-loop,

$$
(y x) C(z, y)=(y x) C\left(z^{-1}, x\right) .
$$

Proof. Let $a=(y x) z^{-1}$. Then

$$
\begin{array}{rlrl}
(y x) C(z, y) & =(y x) C\left(z^{-1}, y^{-1}\right) & & \text { by }(3.3) \\
& =(y x) R\left(z^{-1}\right) L\left(y^{-1}\right) R(z) L(y) & \\
& =a L\left(y^{-1}\right) R(z) L(y) & & \\
& =\left(y^{-1} a\right) R\left(a^{-1}(y x)\right) L(y) & & \\
& =(y x) L\left(a^{-1}\right) L\left(y^{-1} a\right) L(y) & & \text { by }(2.13) \\
& =(y x) L\left(y, a^{-1}\right) & & \text { by }(3.2) \\
& =(y x) L\left(y^{-1}, a\right) & & \\
& =\left(y a^{-1}\right)(a x)=(y x) R\left(x^{-1}, a^{-1}\right) & & \text { by }(3.2) \\
& =(y x) L\left(x^{-1}, a^{-1}\right) & & \text { by }(2.13) \\
& =(y x) L\left(a^{-1}\right) L(x a) L\left(x^{-1}\right) & & \\
& =x^{-1}(x a \cdot z) & & \\
& =(y x) R\left(z^{-1}\right) L(x) R(z) L\left(x^{-1}\right)=(y x) C\left(z^{-1}, x\right) .
\end{array}
$$


Proof of Theorem [1. For $x, y, z \in L$, we compute

$$
\begin{aligned}
x(y(x z)) & =x R(z) L(y) L(x) & & \\
& =x C(z, y) L(y) R(z) L(x) & & \\
& =(y x) C(z, y) R(z) L(x) & & \text { by }(2.11) \\
& =(y x) C\left(z^{-1}, x\right) R(z) L(x) & & \text { by }(3.4) \\
& =(y x) R(z) C\left(z^{-1}, x\right) L(x) & & \text { by }(2.11) \\
& =(y x) L(x) R(z)=(x y x) z . & &
\end{aligned}
$$

Corollary 4. $C(x, z)=L(z, x)=R(z, x)$, and $C(x, z)^{3}=I$.

Proof. By the Moufang equation, $R(x z) L(x)=R(x) L(x) R(z)$. Hence,

$$
R\left(x^{-1}\right) R(x z) R\left(z^{-1}\right)=L(x) R(z) L\left(x^{-1}\right) R\left(z^{-1}\right),
$$

so that (by (2.12) and (2.7) $) R\left(z^{-1}, x^{-1}\right)=C\left(x^{-1}, z^{-1}\right)$. Now use Corollary 3

\section{COMputer-AIDED PROOFS}

We comment further on our use of McCune's program OTTER [12]. This is a general-purpose automated reasoning program which will prove theorems from axioms in first-order logic. In comparison with human reasoning, it is strongest in equational reasoning, and weakest in domains such as set theory, where there are many propositional connectives and alternations of quantifiers. Thus, most of the new mathematics to come out of automated reasoning has been in fields close to algebra. The book by Wos and Pieper [19] describes general methods for applying automated reasoning to problems in mathematics and other areas. Many new theorems proved by OTTER occur in the book by McCune and Padmanabhan 13.

Many authors (as in [13]) simply use the OTTER output as the proof of a theorem. This is mathematically sound, since although OTTER's search procedure is rather complex, the program can be made to output a simple proof object, which can be independently verified by a short lisp program. However, OTTER's proofs are often long sequences of complicated equations which carry little intuitive content, and it is useful to re-express them in a form which a human reader can easily understand and verify.

Some discussion of the procedure for "humanizing" proofs occurs in [6]. This was applied in the case of loop theory in [7, 8, 9, 10, 11, and in the present paper, where much of the argument is cast in the spirit of Bruck and Paige [2], emphasizing grouptheoretic properties of the $R(x)$ and $L(x)$, rather than equations in the loop product and inverse. For example, in Corollary 4 , the statement $C(x, z)^{3}=I$ conveys more information to most human readers than does the equivalent equation,

$$
z^{-1}\left(z\left(\left(z^{-1}\left(z\left(\left(z^{-1}\left(z(y x) x^{-1}\right)\right) x\right) x^{-1}\right)\right) x\right) x^{-1}\right)=y,
$$

which might (in its ascii form) be a typical line of OTTER output. However, some proofs seem to require direct computations in the loop itself. These proofs, although easy enough to verify by hand, may lack some motivation. The need for such computations probably explains why the results of this paper have not been found before. 


\section{ACKNOWLEDGEMENTS}

We wish to thank Tomaš Kepka for suggesting this problem to us.

\section{REFERENCES}

[1] R.H. Bruck, A Survey of Binary Systems, Springer-Verlag, 1958; third printing, 1971. MR 20:76

[2] R.H. Bruck and L.J. Paige, Loops whose inner mappings are automorphisms, Ann. of Math. (2) 63 (1956) 308-323. MR 17:943b

[3] O. Chein, H.O. Pflugfelder, and J.D.H. Smith (eds.), Quasigroups and Loops: Theory and Applications, Sigma Series in Pure Math. 8, Heldermann Verlag, Berlin, 1990. MR 93g:20133

[4] A. Drapal, A-loops close to code loops are groups, Comm. Math. Univ. Carolin. 41 (2000), no. 2, 245-249. CMP 2001:01

[5] T.S.R. Fuad, J.D. Phillips, and X.R. Shen, On diassociative A-loops, submitted.

[6] J. Hart and K. Kunen, Single axioms for odd exponent groups, J. Automated Reasoning 14 (1995) 383-412. MR 96h:68178

[7] K. Kunen, Moufang quasigroups, J. Algebra 183 (1996) 231-234. MR 97f:20096

[8] K. Kunen, Quasigroups, loops, and associative laws, J. Algebra 185 (1996) 194-204. MR 97g:20083

[9] K. Kunen, Alternative loop rings, Communications in Algebra 26 (1998) 557-564. MR 99a:17032

[10] K. Kunen, G-loops and permutation groups, J. Algebra 220 (1999) 694-708. MR 2000j:20133

[11] K. Kunen, The structure of conjugacy closed loops, Transactions Amer. Math. Soc. 352 (2000) 2889-2911. MR 2000j:20132

[12] W.W. McCune, OTTER 3.0 Reference Manual and Guide, Technical Report ANL-94/6, Argonne National Laboratory, 1994; or see: http://www-fp.mcs.anl.gov/division/software/

[13] W. McCune and R. Padmanabhan, Automated Deduction in Equational Logic and Cubic Curves, Lecture Notes in Comp. Sci. \#1095, Springer, Berlin, 1996. MR 98m:68238

[14] R. Moufang, Zur Struktur von Alternativkörpern, Math. Ann. 110 (1934) 416-430.

[15] H. Orlik-Pflugfelder, A special class of Moufang loops, Proc. Amer. Math. Soc. 26 (1970) 583-586. MR 42:407

[16] J.M. Osborn, A theorem on A-loops, Proc. Amer. Math. Soc. 9 (1958) 347-349. MR 20:79

[17] H.O. Pflugfelder, Quasigroups and Loops: Introduction, Sigma Series in Pure Math. 7, Heldermann Verlag, Berlin, 1990. MR 93g:20133

[18] J.D. Phillips, On Moufang A-loops, Comm. Math. Univ. Carolin. 41 (2000), no. 2, 371-375. CMP 2001:01

[19] L. Wos and G. W. Pieper, A Fascinating Country in the World of Computing - Your Guide to Automated Reasoning, World Scientific, 1999.

Department of Mathematics \& Computer Science, Indiana University, South Bend, INDIANA 46634

E-mail address: mkinyon@iusb.edu

URL: http://www.iusb.edu/〜mkinyon

Current address: Department of Mathematics, Western Michigan University, Kalamazoo,

Michigan 49008-5248

Department of Mathematics, University of Wisconsin, Madison, Wisconsin 57306

E-mail address: kunen@math.wisc.edu

$U R L:$ http://www.math.wisc.edu/ kunen

Department of Mathematics, Saint Mary's College of California, Moraga, CaliforNIA 94575

E-mail address: phillips@stmarys-ca.edu

Current address: Department of Mathematics and Computer Science, Wabash College, Crawfordsville, Indiana 47933 